\title{
Magnetospheric period oscillations of Saturn's bow shock
}

\author{
K. E. Clarke, ${ }^{1}$ D. J. Andrews, ${ }^{1}$ A. J. Coates, ${ }^{2,3}$ S. W. H. Cowley, ${ }^{1}$ and A. Masters ${ }^{2,3}$ \\ Received 4 December 2009; revised 1 February 2010; accepted 9 March 2010; published 6 May 2010.
}

[1] Using magnetic field and plasma electron data from 35 Cassini orbits on which the spacecraft crossed Saturn's magnetopause and bow shock during 2004-2007, we provide first evidence for bow shock oscillations related to oscillations in the magnetic field and plasma inside the magnetosphere near the $\sim 11 \mathrm{~h}$ planetary rotation period, termed here the "magnetospheric period." Two case studies are presented showing both bow shock and magnetopause oscillations on given spacecraft passes, together with a statistical study showing that bow shock oscillations observed within a timing window between 0.6 and 1.6 of the magnetospheric period are significantly organized by the phase of the interior field oscillations. Both case and statistical studies indicate that the bow shock and magnetopause oscillate approximately in phase, within a phase uncertainty of about $\pm 25^{\circ}$. The phasing of the oscillations is such that the interior oscillatory field within the magnetospheric "core" region (magnetic shells with equatorial crossing distances less than $\sim 15$ Saturn radii) is rotated $\sim 130^{\circ}-160^{\circ}$ counterclockwise (as viewed from the north) of the instantaneous location of outward boundary excursion maxima. The overall data set suggests that bow shock oscillations are commonly observed over the whole local time range that the spacecraft crossed the boundary ( $\sim 5-17 \mathrm{~h} \mathrm{LT})$ with typical amplitudes of $\sim 1-2 R_{S}$, comparable to results found previously for the corresponding oscillations of the magnetopause. These results thus reveal a new aspect of Saturn's bow shock dynamics, providing first evidence for modulation of its position by the periodic processes observed throughout Saturn's magnetosphere.

Citation: Clarke, K. E., D. J. Andrews, A. J. Coates, S. W. H. Cowley, and A. Masters (2010), Magnetospheric period oscillations of Saturn's bow shock, J. Geophys. Res., 115, A05202, doi:10.1029/2009JA015164.

\section{Introduction}

[2] Despite the fact that Saturn's internally generated magnetic field is closely symmetric about the planet's spin axis [e.g., Dougherty et al., 2005; Burton et al., 2009], strong oscillatory phenomena near the planetary rotation period are nevertheless observed throughout the magnetosphere. These include "strobe-like" variations in the power of Saturn kilometric radiation (SKR) emitted by auroral electrons, together with a rotating pattern of perturbations in the magnetic field and plasma particle fluxes that results in oscillations at a given position with a closely similar synodic period [e.g., Espinosa et al., 2003; Gurnett et al., 2005; Carbary et al., 2007a, 2008; Andrews et al., 2008; Southwood and Kivelson, 2007; Burch et al., 2009; Provan et al., 2009]. In particular, within the quasi-dipolar "core" region of the magnetosphere, on magnetic shells with

\footnotetext{
${ }^{1}$ Department of Physics and Astronomy, University of Leicester, Leicester, UK

${ }^{2}$ Mullard Space Science Laboratory, University College London, Dorking, UK.

${ }^{3}$ Centre for Planetary Sciences at UCL/Birkbeck, London, UK.

Copyright 2010 by the American Geophysical Union. 0148-0227/10/2009JA015164
}

equatorial distances lying within $\sim 15 \mathrm{R}_{\mathrm{S}}$ of the planet $\left(\mathrm{R}_{\mathrm{S}}\right.$ is Saturn's radius equal to $60,268 \mathrm{~km}$ ), the equatorial oscillatory field takes the form of a quasi-uniform field of a few nT amplitude that rotates in the same sense as the planet. The SKR power is found to peak when this field points radially outward in the postmidnight sector. Evidence for outward radial propagation of the field and plasma effects has also been found in these data, such that the oscillation "phase fronts" spiral slowly outward from the planet [Cowley et al., 2006; Carbary et al., 2007b; Andrews et al., 2010]. It has also been found that the period of these oscillations is not strictly constant, but varies by $\sim 1 \%$ on few year time scales [e.g., Galopeau and Lecacheux, 2000; Kurth et al., 2008]. The oscillations cannot therefore be tied directly to the rotation period of the planet, and are hence referred to here as "magnetospheric period" oscillations. It has also recently been discovered that the SKR power modulations occur at somewhat differing periods in the two hemispheres, at $\sim 10.6 \mathrm{~h}$ for northern sources and $\sim 10.8 \mathrm{~h}$ for southern sources during the recent epoch investigated using data from the Cassini orbiter [Kurth et al., 2008; Gurnett et al., 2009]. However, the near-equatorial data examined to date, including those discussed here, clearly relate to the longer southern period, corresponding to the summer hemisphere at Saturn over the interval considered (2004-2007). 
[3] It has been found that the magnetopause also undergoes oscillations near the magnetospheric period, as a likely consequence of oscillations in the total pressure inside the magnetosphere that are related to the field and plasma oscillations. Initial evidence was found in Pioneer 11 flyby data by Espinosa et al. [2003], while Clarke et al. [2006] found further examples in Cassini data that were clearly related to oscillations in the interior field and plasma. Most recently Clarke et al. [2010] have presented a detailed study of magnetopause oscillations observed in Cassini data over the interval 2004-2007. This study demonstrated that oscillatory boundary motions selected within a broad "timing window" centered on the magnetospheric period are related to boundary oscillations at the magnetospheric period by showing that their phasing is closely related to the phase of the interior field oscillations determined by Provan et al. [2009]. The variability in the observed times between successive like crossings of the boundary (e.g., from magnetosphere to magnetosheath) is due both to the finite speed of spacecraft motion across the layer where the oscillation takes place, and to the effect of other phenomena (such as fluctuations in solar wind dynamic pressure and surface waves) that can also affect the boundary position. The results presented by Clarke et al. [2010] demonstrate that magnetospheric period oscillations are commonly observed over the whole range of local times at which the magnetopause was encountered by the spacecraft ( $\sim 03-17 \mathrm{~h} \mathrm{LT})$, and have a phase such that the maximum outward displacement of the boundary occurs when the oscillatory field within the "core" region points approximately away from the boundary. However, the phase was also found to depend on radial distance to the boundary (hence also on local time), such that when this effect is taken into account, outward excursions of the boundary are found to map back along spiral phase fronts into both the rotating plasma "bulge" in the outer magnetosphere reported by Burch et al. [2009] and the plasma density maximum in the inner Enceladus plasma torus observed by Gurnett et al. [2007]. Estimates of the magnetopause oscillation amplitude based on calculations of the spacecraft displacement normal to the boundary while within the oscillation layer yields typical values of $\sim 1-2 \mathrm{R}_{\mathrm{S}}$. Evidence was also presented by Clarke et al. [2010] for the occasional occurrence of extreme amplitudes of $\sim 4-5 \mathrm{R}_{\mathrm{S}}$.

[4] If such oscillations of the magnetopause are commonly present, it seems reasonable to suppose that magnetospheric period oscillations of the bow shock must also occur, since the magnetopause represents the effective obstacle around which the solar wind flows. The bow shock is a consequence of the interaction between the supersonic solar wind and the magnetospheric obstacle, such that the positions of the bow shock and magnetopause boundaries should be directly related. Thus the oscillatory motion of the magnetopause discussed by Clarke et al. [2006, 2010] should produce similar motion of the shock surface. However, to date no evidence has been presented for such bow shock oscillations. In this report we examine Cassini observations of the bow shock during 2004-2007 and show that such oscillations are indeed commonly present. We also provide evidence that their phase and amplitude are com- parable with those of the corresponding magnetopause oscillations.

\section{Data Set Employed}

[5] This study uses Cassini data from Saturn Orbit Insertion (SOI) in July 2004 to Revolution (Rev) 55 in late December 2007, this being the interval for which Provan et al. [2009] determined the phase of the "core" region magnetic field oscillations. It is also the interval examined by Clarke et al. [2010] for magnetopause oscillations. The orbits used here divide into two separate intervals, the first of which involves bow shock crossings at low planetary latitudes in the morning sector (SOI to Rev 21), while the second involves crossings in the postnoon to dusk sector (Revs 40 to 55), some of which occur at latitudes significantly away from the equator. The gap between these intervals corresponds mainly to Revs for which apoapsis was located in the nightside sector, and is wider than that in the corresponding magnetopause study since a small number of orbits near the "ends" of the gap cross the magnetopause but do not achieve sufficient radial ranges to also encounter the bow shock. Data gaps necessitated the exclusion of one orbit and affected several others to varying degrees without rendering them wholly unusable. In all, 35 Cassini orbits involving crossings of the bow shock are used in this study.

[6] Crossings of the bow shock and magnetopause were identified in this study by examination of magnetic field data from the Cassini fluxgate magnetometer [Dougherty et al., 2004], together with thermal electron data from the CAPS/ELS electron spectrometer [Young et al., 2004]. The electron data cover the energy range from $0.6 \mathrm{eV}$ to $26 \mathrm{keV}$, thus spanning the usual range of electron energies in the solar wind (typically a few $\mathrm{eV}$ ), magnetosheath (tens of $\mathrm{eV}$ to $\sim 100 \mathrm{eV})$, and outer magnetosphere $(\sim 100 \mathrm{eV}$ to a few $\mathrm{keV})$ at Saturn. Data averaged at $1 \mathrm{~min}$ resolution were employed from both instruments.

\section{Equatorial Magnetic Field Phase Model}

[7] As in the study of magnetopause oscillations presented by Clarke et al. [2010], this report investigates the phasing of the ensemble of Cassini bow shock transitions with respect to the "Provan phase" describing the magnetic field oscillations within the magnetospheric "core" [Provan et $a l ., 2009]$. This phase is given by

$$
\Psi_{M c}(\varphi, t)=\Phi_{M}(t)-\varphi
$$

where $\varphi$ is azimuth measured from noon positive toward dusk, and $\Phi_{M}(t)$ is a near-linearly increasing function of time $t$ (defined by a fifth-order polynomial; see Clarke et al. [2010] and Provan et al. [2009] for details), that determines the slowly varying period $\tau_{M}(t)$ of the oscillations through $\tau_{M}(t)=360 /\left(d \Phi_{M} / d t\right)$ if $\Phi_{M}$ is in degrees. We note that the Provan phase $\Psi_{M c}$ is approximately related to the SLS3 "longitude" system of Kurth et al. [2008] by

$$
\lambda_{S K R}(\varphi, t) \approx \Psi_{M c}(\varphi, t)+250^{\circ},
$$

though $\Psi_{M c}$ is tied directly to the observed magnetic field oscillations rather than to SKR power modulations. Specif- 


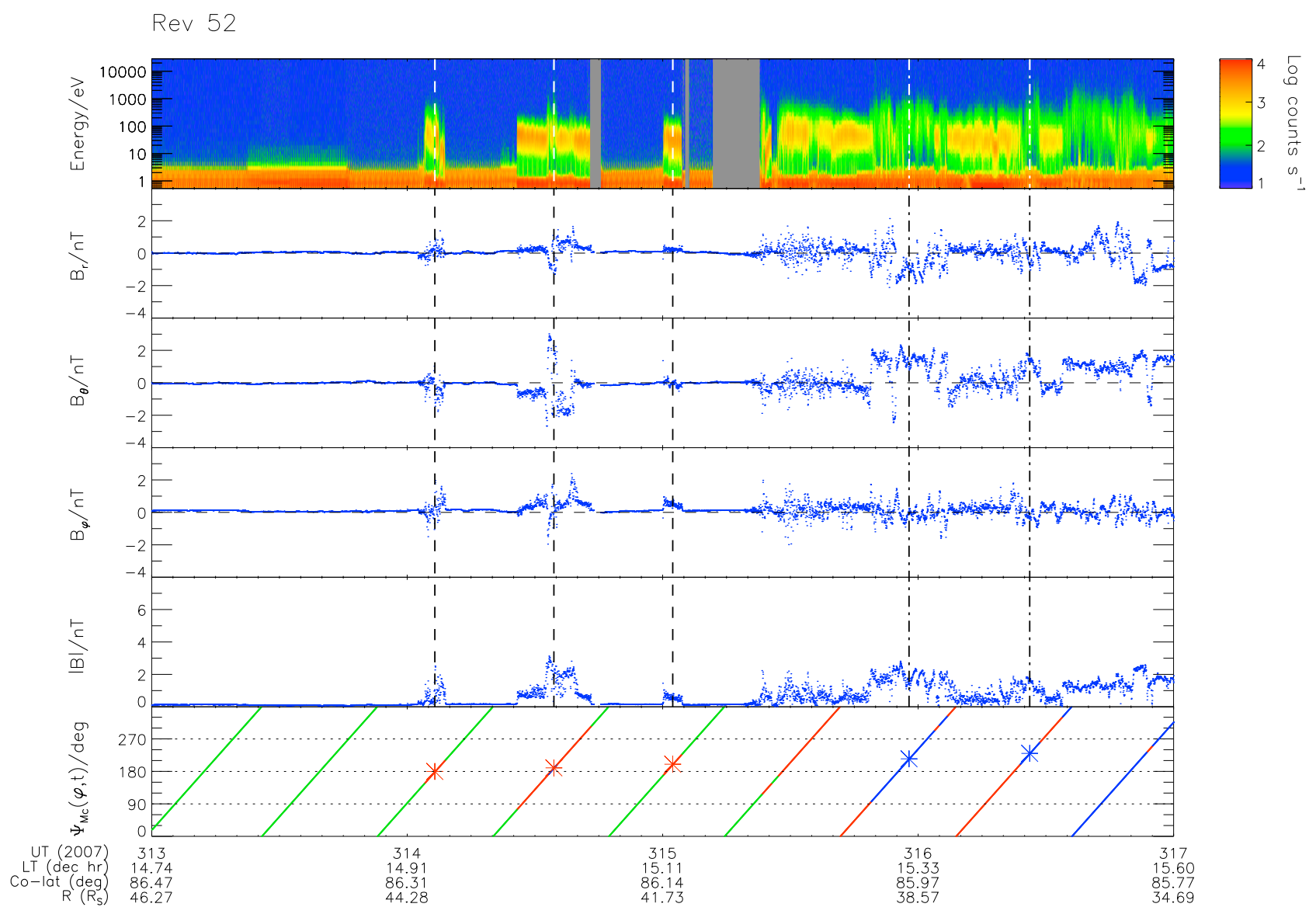

Figure 1. Electron count rate spectrogram, magnetic field data, and Provan phase (modulo $360^{\circ}$ ) for the inbound pass of Cassini Rev 52, days 313-316 of 2007.

ically, the magnetospheric period perturbations in the spherical polar radial $(r)$, meridional $(\theta)$, and azimuthal $(\varphi)$ components of the magnetic field, referenced to the planet's spin/magnetic axis, vary in the near-equatorial "core" region as

$$
B_{r, \theta}=B_{0 r, \theta} \cos \left(\Psi_{M c}\right) \quad B_{\varphi}=B_{0 \varphi} \sin \left(\Psi_{M c}\right),
$$

such that the field variations rotate around the planet in the sense of planetary rotation as a " $m=1$ " disturbance (where $m$ is the azimuthal wave number), with synodic period $\tau_{M}$. We note that in the case of equal amplitudes, $B_{0 r}=B_{0 \varphi}$, the equatorial radial and azimuthal field components combine to form a quasi-uniform rotating field as described in section 1. At time $t$ this field is directed at an azimuth relative to the Sun given by

$$
\varphi_{M}(t)=\Phi_{M}(t)
$$

again measured positive from noon toward dusk.

\section{Examples of Bow Shock Oscillations}

[8] Transitions across the bow shock from the upstream solar wind to the magnetosheath are readily recognized in the combined magnetic field and thermal electron data by sudden simultaneous increases in the electron flux in the range $10-100 \mathrm{eV}$ and in the field strength. Transitions across the magnetopause from the magnetosheath to the magnetosphere are similarly characterized by substantial reductions in the electron flux in the $10-100 \mathrm{eV}$ range and increases at $100 \mathrm{eV}$ to $1 \mathrm{keV}$, along with increases in the field strength, generally accompanied by changes in direction to southward orientations and reductions in variability. Visual inspection of the data is sufficient to detect many examples of magnetopause oscillations near the magnetospheric period, which analysis shows are closely related to the phase of the magnetospheric field oscillations as outlined in section 1 [Clarke et al., 2010]. Overall, the behavior of the bow shock appears rather more variable, so that oscillations near the magnetospheric period are generally less obvious. Nevertheless, such oscillatory behavior is evident on a number of passes, two of which are presented here as examples.

[9] Figure 1 shows 4 days of data (days 313-316 of 2007) from the inbound pass of Rev 52. The first panel shows an electron count rate spectrogram from anode 5 of the ELS instrument, color-coded according to the scale on the right. The second through fifth panels show 1 min averaged values of the spherical polar components of the magnetic field referenced (as above) to the planet's spin/magnetic axis, together with the magnitude of the field. The sixth panel shows the phase of the "core" magnetic field oscillations $\Psi_{M c}$ (modulo $360^{\circ}$ ) given by equation (1), evaluated at the spacecraft azimuth $\varphi_{s}(t)$. The phase values are color-coded 
according to whether the spacecraft is situated in the solar wind (green), magnetosheath (red), or magnetosphere (blue). We note that since we can write $\Psi_{M c}\left(\varphi_{s}(t), t\right)=\varphi_{M}(t)-\varphi_{s}(t)$ from equations (1) and (4), the value of $\Psi_{M c}$ gives the instantaneous angle of the model magnetic field within the "core" region with respect to the azimuth of the spacecraft (positive counterclockwise viewed from the north, as for the definition of azimuth $\varphi$ ). Spacecraft position data, specifically local time, colatitude (again with respect to the spin/ magnetic axis), and radial distance from the planet's center, are given at the bottom of the plot. The pass takes place in the midafternoon sector, at $\sim 15 \mathrm{~h} \mathrm{LT}$, near the planet's equatorial plane, with the data shown in Figure 1 spanning a radial range from 46.3 to $34.7 \mathrm{R}_{\mathrm{S}}$.

[10] At the beginning of the interval the spacecraft is located in the solar wind, as is evident from the weak thermal electron fluxes (the intense fluxes at and below a few $\mathrm{eV}$ in the spectrogram are principally spacecraft photoelectrons), and the very small field strengths of $\sim 0.2 \mathrm{nT}$. Shortly after the start of day 314 there begins a sequence of three quasiperiodic magnetosheath encounters, the temporal separations between the midpoints of which are $\sim 11 \mathrm{~h}$, thus being close to the magnetospheric period. The vertical dashed lines in the first through fifth panels mark the midpoints of the encounters, while the stars in the sixth panel show the corresponding Provan phase $\Psi_{M c}$ at the midpoints, which is near-constant at $\sim 180^{\circ}$. A Provan phase value of $\sim 180^{\circ}$ means that the "core" equatorial field points approximately away from the spacecraft at the center times of the magnetosheath encounters, these center times representing proxies for the times of maximum radial outward displacement of the bow shock. We note that this phasing is essentially the same as that found previously for the maximum outward displacement of the magnetopause [Clarke et al., 2010]. The final crossing into the magnetosheath takes place at $\sim 10 \mathrm{~h}$ UT on day 315 near to the time of the next expected encounter (when the Provan phase is again $\sim 180^{\circ}$ ), following which two quasiperiodic encounters with the magnetosphere are observed, also separated by $\sim 11 \mathrm{~h}$ as marked by the vertical dot-dashed lines. These have slightly later phases of $215^{\circ}$ and $230^{\circ}$, which nevertheless lie well within the usual scatter of magnetopause phase values at this radial distance and local time [Clarke et al., 2010]. After $\sim 14$ h UT on day 316 the spacecraft finally remains within the magnetosphere on the pass, except for one brief ( $30 \mathrm{~min})$ excursion into the magnetosheath toward the end of day 316.

[11] The data from this Rev provide good evidence of approximately in-phase magnetospheric period oscillations of the bow shock and magnetopause on the same pass, which in this case appear to be of considerable amplitude. Assuming that the observed boundary motions are associated wholly with the magnetospheric period oscillations, the radial displacement of the spacecraft between the first bow shock encounter on day 314 and the last on day 315 represents a lower limit for twice the oscillation amplitude. This displacement is $\sim 5 \mathrm{R}_{\mathrm{S}}$, thus suggesting an amplitude of $\sim 2.5 \mathrm{R}_{\mathrm{S}}$ or more. Indeed, careful inspection of the data during the second quasiperiodic magnetosheath encounter reveals that the spacecraft briefly entered the magnetosphere at the center of the oscillation, indicating that the boundary motion was sufficient in this case to displace both the bow shock and the magnetopause temporarily across the spacecraft.
Since the radial separation of the bow shock and magnetopause is typically $\sim 9 \mathrm{R}_{\mathrm{S}}$ under the conditions pertaining here [Arridge et al., 2006; Masters et al., 2008], the implied peak-to-peak displacement of the boundary during this particular oscillation must be of comparable magnitude. It may be noted that previous examples of particularly large-amplitude oscillations of the magnetopause were all observed, as here, in the postnoon sector [Clarke et al., 2010]. We also note that the central location of the brief magnetosphere encounter within the transient magnetosheath interval again suggests that the two boundaries oscillate approximately in phase.

[12] The second example in Figure 2 shows 9 days of data (days 116-124 of 2007) spanning the apoapsis pass of Revs 43 outbound and 44 inbound. Apoapsis itself occurs at a radial distance of $36.2 \mathrm{R}_{\mathrm{S}}$ at $\sim 23 \mathrm{~h} \mathrm{UT}$ on day 122, at $\sim 16 \mathrm{~h} \mathrm{LT}$. During the first 2 days of the interval the spacecraft passes out from the magnetosphere, across the magnetosheath into the solar wind in the near-equatorial postnoon sector. A number of short-period magnetopause and bow shock encounters are observed, reminding us that the positions of these boundaries are subject to a range of phenomena (e.g., variations in solar wind parameters [Arridge et al., 2006; Masters et al., 2008], magnetic reconnection at the magnetopause [McAndrews et al., 2008], and Kelvin-Helmholtz waves at the magnetopause [Masters et al., 2009]) in addition to the longer-period oscillations studied here. During the approach to apoapsis over the following 4 days, however, a quasiperiodic sequence of magnetosheath encounters near the magnetospheric period are observed, marked by the vertical dashed lines. A sequence of three such encounters occurs on days 118-119, and following one "missed" encounter due possibly to solar wind variations, a further sequence of three encounters takes place on days 120 121. A fourth encounter in the latter sequence is obscured by the data gap beginning near the end of day 121 . The Provan phases of these encounters, shown by the red stars in the sixth panel, are more scattered than in the previous example, between $\sim 90^{\circ}$ and $\sim 210^{\circ}$, but the six values are still grouped within a band of $\sim 120^{\circ}$ of phase (which also contains the magnetosheath encounter phases in Figure 1), avoiding the remaining $\sim 240^{\circ}$ of phase. After the data gap the spacecraft passes once more between the solar wind and the magnetosheath, and then observes a sequence of four quasiperiodic encounters with the magnetosphere, again marked by the vertical dot-dashed lines. The Provan phases of these magnetosphere encounters are seen to occupy a similar but slightly broader range of phase to those of the magnetosheath encounters $\left(\sim 80^{\circ}-230^{\circ}\right)$, but still lying within the usual range of magnetopause phase values at this local time [Clarke et al., 2010].

\section{Relation to Magnetic Phase in the "Core" Region}

[13] While the examples in Figures 1 and 2 provide case study evidence for the occurrence of magnetospheric period oscillations of the bow shock related to comparable oscillations of the magnetopause, the evidence in the data from most passes through the boundary region is much less clearcut, consisting mainly of single observed oscillations of the boundaries (if any at all). The approach taken in the related 
Rev $43 / 44$

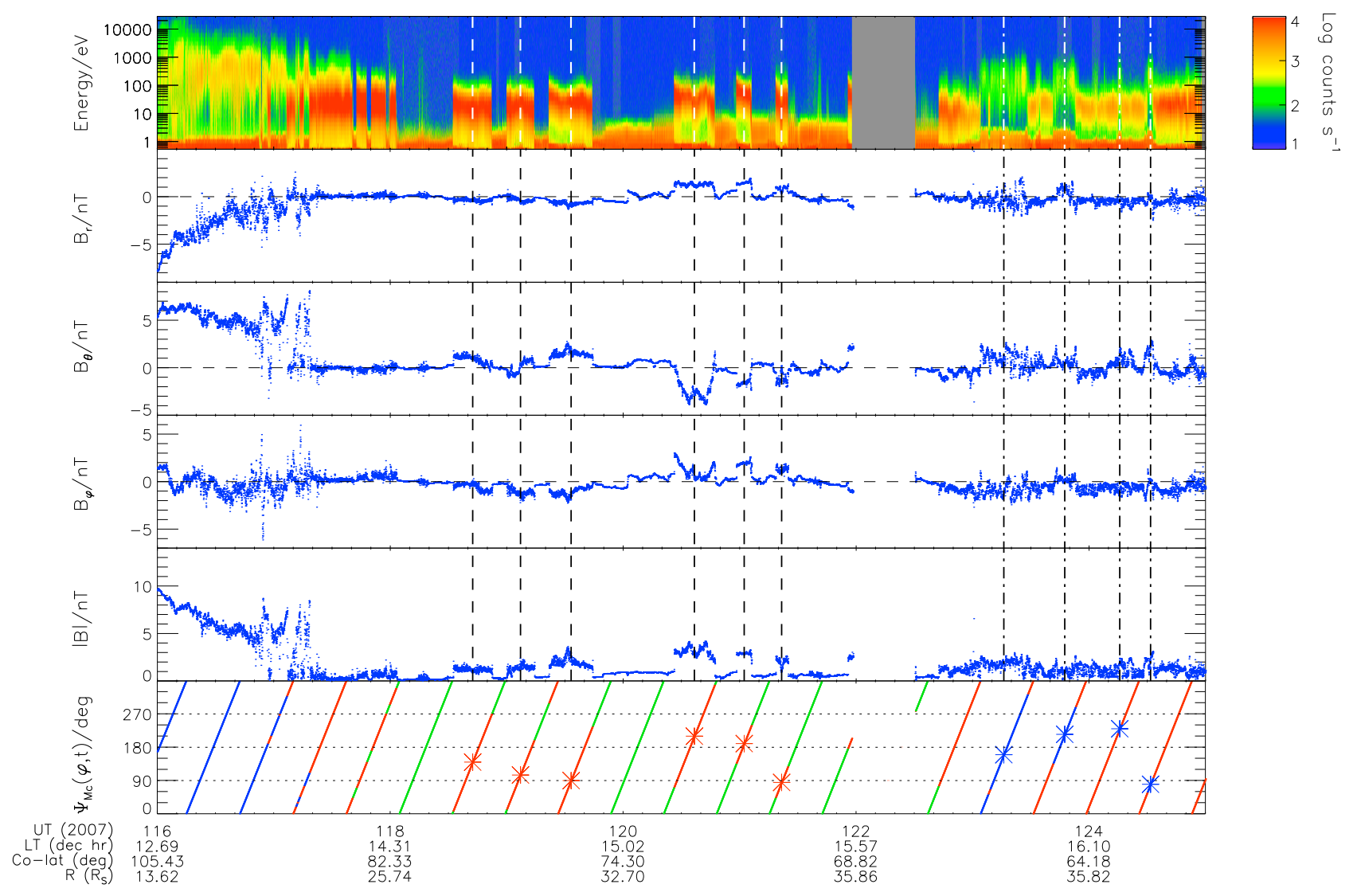

Figure 2. Electron count rate spectrogram, magnetic field data, and Provan phase (modulo $360^{\circ}$ ) for Cassini Rev 43 outbound and 44 inbound, days 116-124 of 2007.

study of magnetopause oscillations by Clarke et al. [2010] was to apply a "timing window" to the interval between successive like crossings of the boundary to select those crossings near the magnetospheric period, and then to examine whether this subset of crossings is related in phase to that of the magnetic field oscillations within the magnetospheric "core." Motion of the spacecraft through the boundary region at a finite speed results in crossing intervals that are always less than the period of the oscillating boundary [Clarke et al., 2010], so initially it might be thought that the timing window should similarly be confined to shorter intervals. It must be recognized, however, that a range of additional phenomena also influence these timings, as noted in section 4. Consequently, a relatively wide timing window between 0.4 and 1.6 of the $\sim 10.8 \mathrm{~h}$ magnetospheric period was employed by Clarke et al. [2010] (i.e., between $\sim 4.3$ and $\sim 17.3 \mathrm{~h}$ ), since it was found that the magnetopause oscillations were significantly organized by the Provan phase over the whole of this timing window band. However, the numbers of oscillations in the data set and the degree of phase organization both decrease toward the ends of the band.

[14] Here we have applied the same technique to the ensemble of bow shock encounters observed by Cassini during Revs SOI-55, to examine the extent to which the phasing of the bow shock motions within various timing windows are related to the Provan phase. The results are somewhat more scattered than for the magnetopause, in line with our comments in section 4. However, phase organization is found to be present throughout the timing band between 0.6 and 1.6 of the magnetospheric period, with the strongest organization being found between 0.6 and 1.0 of that period. In contrast to the results for the magnetopause, however, no phase organization is found between 0.4 and 0.6 of the magnetospheric period. We have thus adopted a timing window for bow shock oscillations between 0.6 and 1.6 of the magnetospheric period for the results discussed below. In order to make a close comparison we have also reanalyzed the magnetopause observations using the same timing window criterion. For the same reason we have also restricted our magnetopause data set to those spacecraft orbits which also crossed the bow shock at some point, thus excluding a number of orbits where the spacecraft crossed the magnetopause, but had insufficient radial range to reach the more distant bow shock, as mentioned previously in section 2 .

[15] The data set consists of 35 orbits during which 62 bow shock oscillations and 71 magnetopause oscillations were observed within the above timing window. We have then determined the Provan phase given by equation (1) for each of these oscillation events, specifically at the center time of the magnetosheath interval for the bow shock oscillations and the magnetosphere interval for the magnetopause oscillations, these being proxies for the time of maximum outward boundary excursion. The results are presented in 


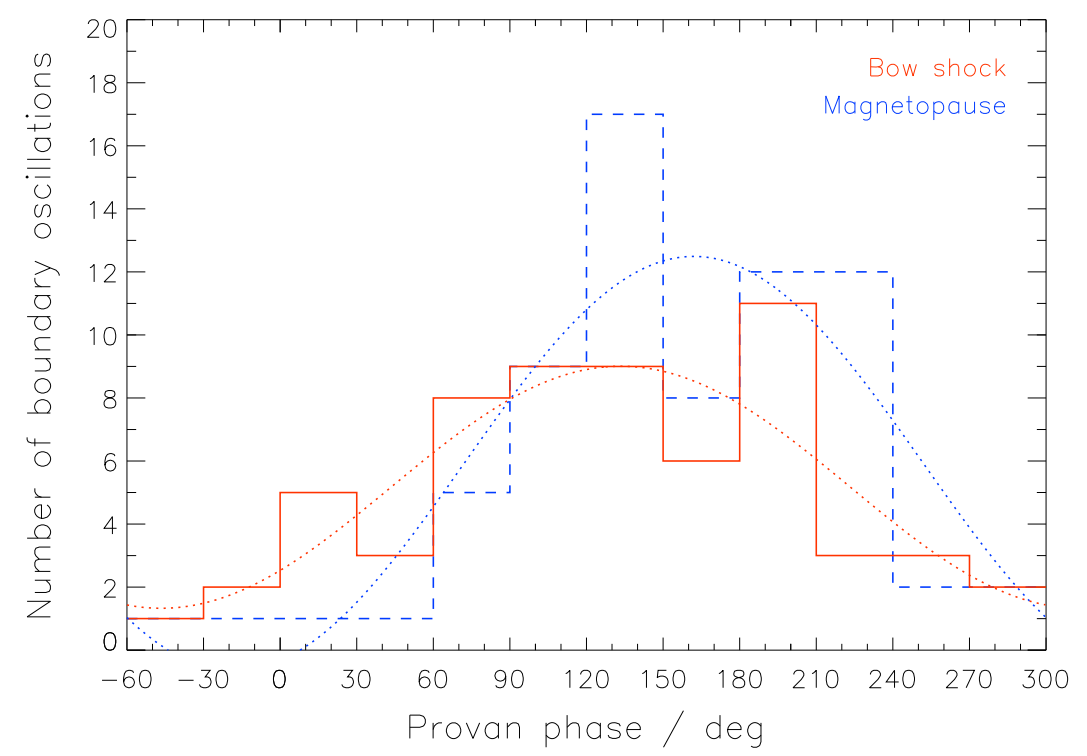

Figure 3. Histograms of the number of observed bow shock (red) and magnetopause (blue) oscillations versus Provan phase (modulo $360^{\circ}$ ) for an event timing window of 0.6 to 1.6 of the magnetospheric period, with least squares sinusoid fits.

Figure 3, where we show histograms of the number of oscillations occurring within $30^{\circ}$ bins of Provan phase, the red line corresponding to the bow shock and the blue line to the magnetopause. It can be seen that both distributions are significantly peaked over similar ranges of Provan phase, demonstrating that both are related to the field and plasma oscillations inside the magnetosphere. It is also evident, however, that the distribution of bow shock phase values is somewhat broader than that of the magnetopause oscillations. If we consider the oscillation events that occur within the preferred $180^{\circ}$ phase sector between $60^{\circ}$ and $240^{\circ}$ of both distributions (a sector that we note contains all of the phase values determined in Figures 1 and 2 for both bow shock and magnetopause), we find that for the bow shock $74 \%$ of the oscillations occur in this sector and $26 \%$ in the other $180^{\circ}$ sector from $240^{\circ}$ to $60^{\circ}$ (via $360^{\circ}$ ), while for the magnetopause $89 \%$ occur in this preferred sector and $11 \%$ in the other sector. If the data set is further restricted in both cases to the timing window between 0.6 and 1.0 of the magnetospheric period, consisting of 41 bow shock oscillations and 40 magnetopause oscillations, we find that the phase organization within the preferred sector is marginally increased, such that $78 \%$ of the bow shock oscillations and $90 \%$ of the magnetopause oscillations then occur in this sector $\left(60^{\circ}-240^{\circ}\right)$. Thus while these results show that the bow shock oscillations within the above timing windows are certainly organized by the Provan phase, hence proving the existence of magnetospheric period oscillations of the bow shock on a statistical basis, the degree of organization is rather less than that found for the magnetopause.

[16] It is of interest to determine and compare the Provan phases $\Psi_{0}$ at which the histograms in Figure 3 peak. To do this we have least squares fitted the histogram values to a sinusoid given by $N=a \cos \left(\Psi_{M c}-\Psi_{0}\right)+b$, which is shown by the appropriately colored curves in Figure 3 . The uncertainty in the phase at the peak has been determined by computing the phase displacement away from the best fit value that results in a significant $10 \%$ increase in the RMS deviation of the fitted line from the histogram data. The resulting phases are $133^{\circ} \pm 17^{\circ}$ for the bow shock and $162^{\circ} \pm 15^{\circ}$ for the magnetopause, the latter being closely similar to the magnetopause value previously determined by Clarke et al. [2010]. The difference between these values is $-29^{\circ} \pm 23^{\circ}$, which is essentially consistent with zero. For the subset of data within the restricted timing window between 0.6 and 1.0 of the magnetospheric period, the peak phase values are found to be $140^{\circ} \pm 19^{\circ}$ for the bow shock and $144^{\circ} \pm 21^{\circ}$ for the magnetopause, again consistent with in-phase behavior within the estimated errors. The physical meaning of these results is that the quasi-uniform "core" magnetic field is typically rotated $\sim 130^{\circ}-160^{\circ}$ counterclockwise (as viewed from the north) from the instantaneous position of outward boundary maxima, thus pointing approximately away from the latter.

[17] To take the comparison a step further, in Figure 4 we show the Provan phase of each observed bow shock (red) and magnetopause (blue) oscillation for the timing window between 0.6 and 1.6 of the magnetospheric period, plotted versus the local time at the center of the oscillation. In Figure 4 we now include data from five orbits that have magnetopause crossings but no bow shock crossings. The data have been divided into three $3 \mathrm{~h}$ local time bands centered on 6,9 , and $15 \mathrm{~h}$ LT where there are significant numbers of data values for both sets of oscillations, and the most probable phase values (with error estimates) have been calculated in the same manner as in Figure 3. These values are shown by the red and blue squares, from which it can be seen that the phase values for the LT bins centered on 6 and $15 \mathrm{~h}$ are equal within the error bars (typically about $\pm 25^{\circ}$ ), while for the bin centered on $9 \mathrm{~h}$ LT the difference lies marginally outside of the error bars. It can also be seen that the two phase values for the bin centered on $6 \mathrm{~h} \mathrm{LT}$ on the dawn meridian both take somewhat larger values than those in the local time bands centered on 9 and $15 \mathrm{~h}$ LT either side 


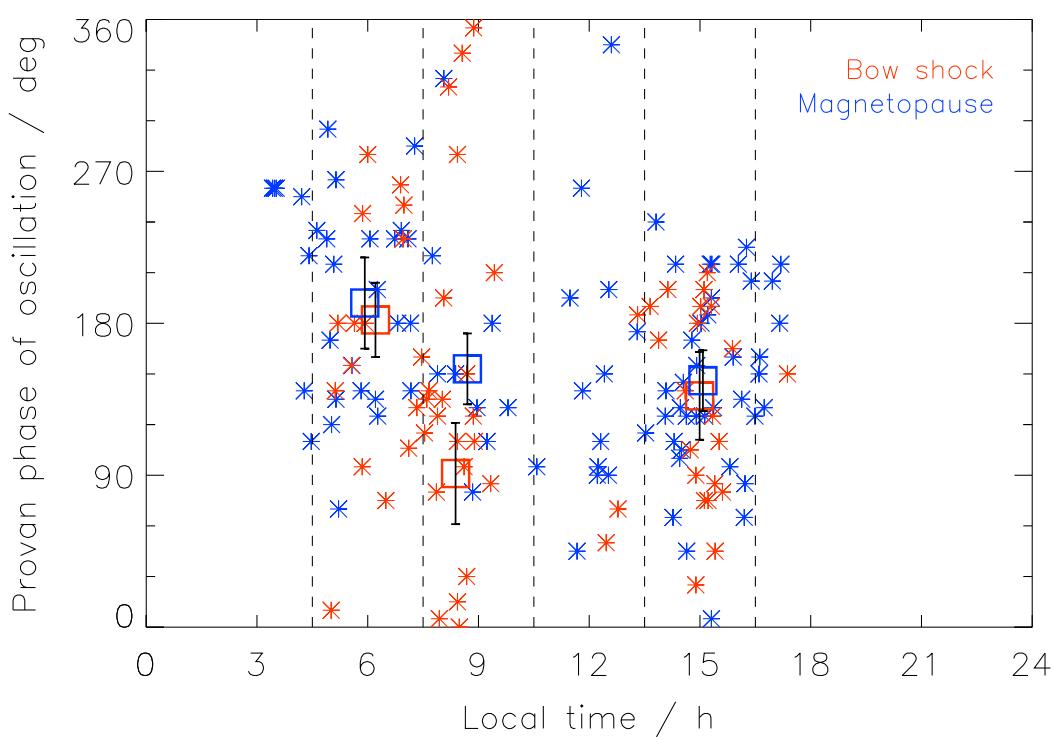

Figure 4. Plot of the Provan phase (modulo $360^{\circ}$ ) of the bow shock (red) and magnetopause (blue) oscillations versus local time for an event timing window of 0.6 to 1.6 of the magnetospheric period. The squares show peak phase values determined by least squares sinusoid fits in the $3 \mathrm{~h}$ windows of LT marked by the vertical dashed lines.

of noon. This is due to the larger radial distance to the boundary with increasing azimuth from noon, combined with the finite outward radial propagation speed of the oscillatory disturbances within the magnetosphere [Clarke et al., 2010].

[18] Overall, the above results demonstrate that the magnetospheric period oscillations of the bow shock are in phase with those of the magnetopause, within a phase uncertainty of about $\pm 25^{\circ}$. This result implies a sufficiently fast propagation of the oscillatory signal in the magnetosheath between the two boundaries. Since the separation of the boundaries in the midmorning and midafternoon LT hours corresponding to the majority of the data examined here (Figure 4) is $\sim 5-10 \mathrm{R}_{\mathrm{S}}$ (see Figure 5, to be introduced in section 6), the implied outward propagation speed for approximately in-phase oscillations within the above phase uncertainty is at least $\sim 100-200 \mathrm{~km} \mathrm{~s}^{-1}$. Physically, for slow motions of the magnetopause boundary as is the case here $\left(\sim 10-30 \mathrm{~km} \mathrm{~s}^{-1}\right.$ as estimated by Clarke et al. [2010]), we expect the signal to propagate through the magnetosheath at the magnetosonic speed. In the near subsolar magnetosheath this speed will be some significant fraction of the upstream solar wind speed (the latter being typically $\sim 500 \mathrm{~km} \mathrm{~s}^{-1}$ ), thus consistent with the lower limits for outward propagation of $\sim 100-200 \mathrm{~km} \mathrm{~s}^{-1}$ estimated above. We note that the corresponding radial propagation speed of these signals in the magnetosphere is estimated to be $\sim 200-400 \mathrm{~km} \mathrm{~s}^{-1}$ [Andrews et al., 2010; Clarke et al., 2010].

\section{Occurrence and Amplitude of Bow Shock Oscillations}

[19] In this section we finally consider some implications of our findings for the occurrence and amplitude of the bow shock oscillations. In the corresponding discussion of magnetopause oscillations by Clarke et al. [2010], it was assumed that all of the oscillations observed within their timing window were associated with boundary oscillations at the magnetospheric period. This assumption was made on the basis that $\sim 90 \%$ of their phase values fell within one "preferred" $180^{\circ}$ sector of Provan phase and only $\sim 10 \%$ in the other, equivalent to the magnetopause result shown here (with modestly different timing window and orbit selection) in Figure 3. However, this argument has rather less force for the bow shock oscillations, since the percentage of observed oscillations whose phase falls within the preferred $180^{\circ}$ sector, between $60^{\circ}$ and $240^{\circ}$, is reduced to $\sim 75 \%$, with $\sim 25 \%$ in the other sector. While the peaking in Provan phase within this sector is sufficient to show the presence of bow shock motion at the magnetospheric period on a statistical basis, it also suggests that not all of the observed oscillations within the timing window are so associated. Of course, it is not possible to say which are and which are not on an individual basis. In order to take the discussion of occurrence and amplitude further, we thus introduce an additional element of event selection to consider only those oscillations that occur within the "preferred" phase sector, on the basis that at least a majority of those cases are likely to be associated with the magnetospheric period oscillation. We apply this rule equally to both bow shock and magnetopause oscillations so that the results may be directly compared, though this produces only a marginal change in the more highly phase-peaked magnetopause data set.

[20] We begin by noting that out of the total of 35 Cassini orbits that pass through the magnetosheath-solar wind boundary region, 22 are found to exhibit bow shock oscillations on either the outbound or inbound passes (falling both within the 0.6-1.6 magnetospheric period timing window and the preferred phase sector between $60^{\circ}$ and $240^{\circ}$ ). Such oscillations are therefore found on $\sim 60 \%$ of these orbits, thus representing a relatively commonly observed phenomenon. Of the 22 orbits exhibiting such oscillations, 8 have one oscillation, 9 two oscillations, and 5 three or more (not necessarily consecutive). On these same 35 orbits, 

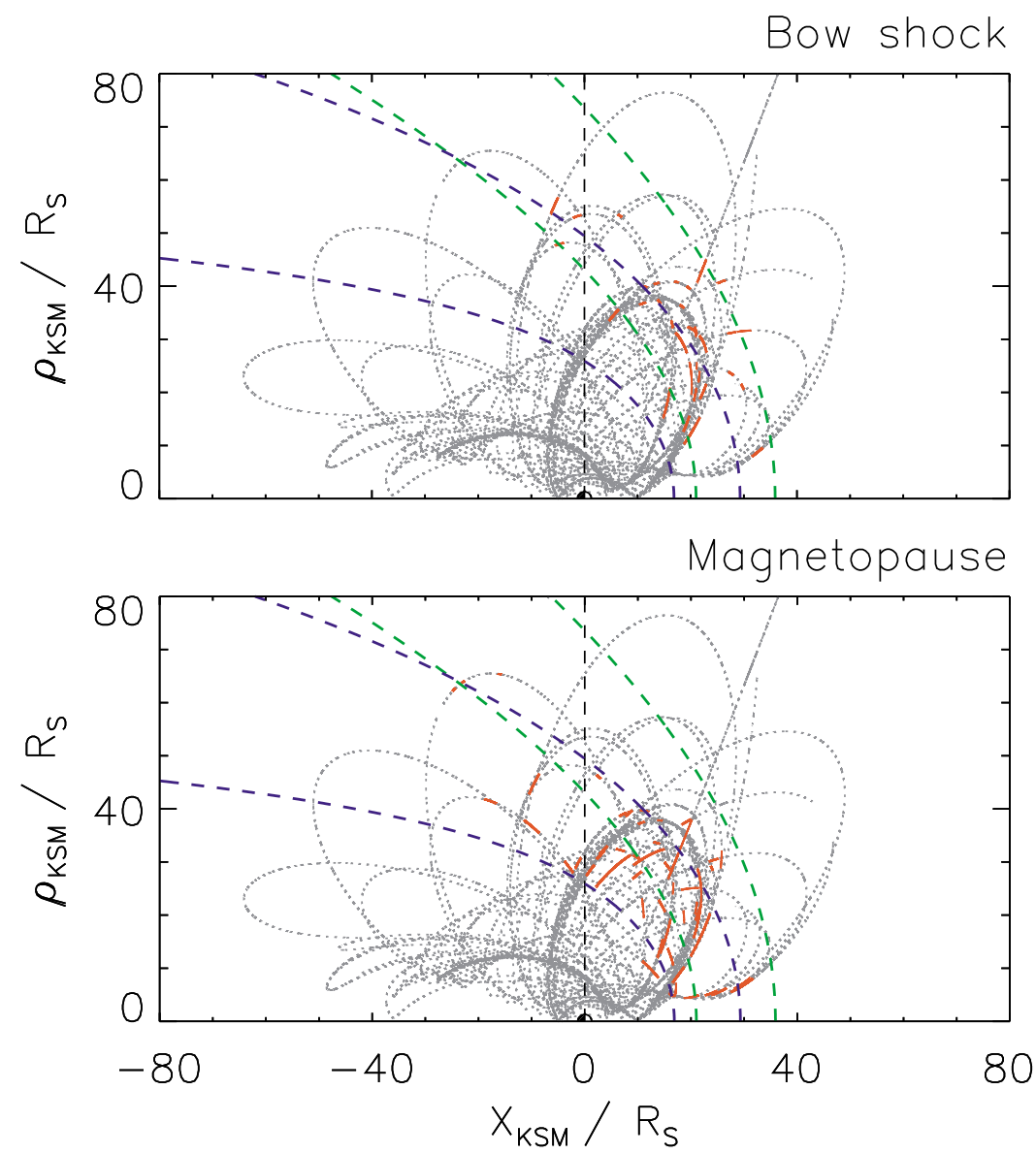

Figure 5. Plots showing the distribution of (top) bow shock and (bottom) magnetopause oscillations (red orbit segments) in cylindrical KSM coordinates for an event timing window of 0.6 to 1.6 of the magnetospheric period and the "preferred" $180^{\circ}$ sector of Provan phase (modulo $360^{\circ}$ ) from $60^{\circ}$ to $240^{\circ}$.

25 exhibit magnetopause oscillations within the same timing window and preferred sector, i.e., $\sim 70 \%$ of the orbits in this case. Of these, 12 have one oscillation, 4 two oscillations, and 9 three or more. Clarke et al. [2010] reported their magnetopause oscillation occurrence results on the basis of individual passes through the magnetopause boundary region, but if analyzed on the same whole-orbit basis as adopted here, their occurrence rises to $\sim 85 \%$. The $\sim 70 \%$ value obtained here thus represents a modest reduction in reported magnetopause oscillation occurrence compared with Clarke et al. [2010], resulting from the somewhat narrower timing window and preferred sector selection employed here, but still showing that it is a common phenomenon. Overall, our results thus show that bow shock oscillations near the magnetospheric period are commonly present, comparable in occurrence to the equivalent oscillations of the magnetopause reported by Clarke et al. [2010].

[21] The spatial distribution of the selected bow shock and magnetopause oscillations is shown in KSM coordinates in Figure 5 (where, as in Figure 4, we show the magnetopause oscillations irrespective of whether the bow shock was crossed on a particular pass). KSM is the preferred system for bow shock and magnetopause studies, with $X_{K S M}$ pointing toward the Sun (approximately opposite to the solar wind flow), the $X_{K S M}-Z_{K S M}$ plane containing the planet's spin and magnetic axis, and $Y_{K S M}$ completing the right- handed triad. The magnetopause and bow shock models of Arridge et al. [2006] and Masters et al. [2008] are expressed as figures of revolution about the $X_{K S M}$ axis, and are plotted in Figure 5 in cylindrical $\rho_{K S M}-X_{K S M}$ coordinates, where $\rho_{K S M}=\sqrt{Y_{K S M}^{2}+Z_{K S M}^{2}}$, by the blue and green dashed lines, respectively. The inner pair of lines correspond to a solar wind dynamic pressure of $0.1 \mathrm{nPa}$, and the outer pair to 0.01 nPa, spanning the usual range [e.g., Arridge et al., 2006; Achilleos et al., 2008]. The trajectory of the spacecraft is shown by the gray dashed lines in the same cylindrical coordinates, plotted over the full tour employed here (SOI to Rev 55). Red segments of the trajectory correspond to boundary oscillations of the bow shock (Figure 5, top) and the magnetopause (Figure 5, bottom), and specifically show the whole of each $0^{\circ}-360^{\circ}$ rotation of the Provan phase containing an observed oscillation, so that oscillations that are consecutive are shown by a continuous red line. It can be seen that both bow shock and magnetopause oscillations are observed over essentially the whole range of locations that the spacecraft orbit crossed the expected position of these boundaries. This range corresponds to local times of 5-17 $\mathrm{h}$ for the bow shock and 3-17 h LT for the magnetopause (as also seen in Figure 4), and also to KSM latitudes between $-18^{\circ}$ and $+32^{\circ}$ for the bow shock and $-36^{\circ}$ and $+43^{\circ}$ for the magnetopause. 


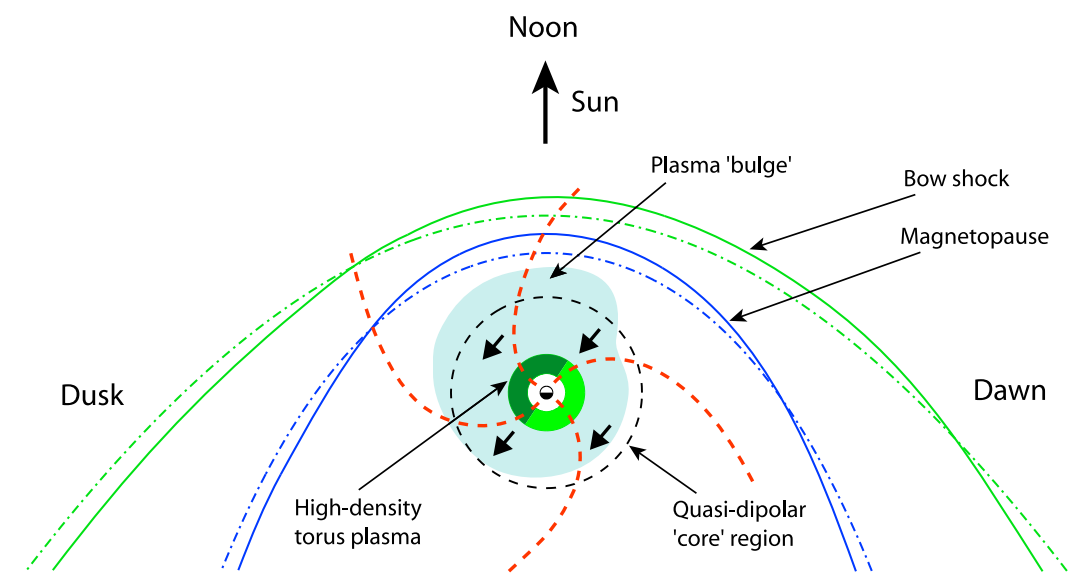

Figure 6. Sketch showing the relationships between various oscillatory phenomena in Saturn's equatorial magnetosphere, viewed from the north with noon at the top and dusk to the left. At the instant shown, the maximum outward displacements of the magnetopause and bow shock occur near the noon meridian.

[22] Finally with regard to the amplitude of the oscillations, we note with Clarke et al. [2010] that although estimates are difficult, a lower limit to the peak-to-peak amplitude can be estimated from the spacecraft displacement normal to the model boundary surfaces between first and last contacts with the oscillating boundary (assuming that the boundary position is not affected by other processes during the interval in question). For our selected cases in which only a single boundary oscillation was observed ( 22 cases for the bow shock and 26 for the magnetopause), the mean displacements are found to be $0.9 \mathrm{R}_{\mathrm{S}}$ for the bow shock and $1.8 \mathrm{R}_{\mathrm{S}}$ for the magnetopause. With regard to the nature of the lower limit, the theoretical analysis presented by Clarke et al. [2010, Figure $2 \mathrm{~d}$ and Table 1] suggests that the displacement for single oscillations corresponds to roughly half the peak-to-peak boundary displacement, such that our results imply amplitudes of $\sim 0.8 \mathrm{R}_{\mathrm{S}}$ for the bow shock and $\sim 1.6 \mathrm{R}_{\mathrm{S}}$ for the magnetopause, the magnetopause result being consistent with Clarke et al. [2010]. Thus both results imply typical amplitudes of order $\sim 1 \mathrm{R}_{\mathrm{S}}$, the significance of the difference between them remaining unclear. Correspondingly, for our selected cases in which two consecutive boundary oscillations were observed ( 6 cases for the bow shock and 8 for the magnetopause), the mean spacecraft displacements are found to be $2.5 \mathrm{R}_{\mathrm{S}}$ for the bow shock and 3.0 $\mathrm{R}_{\mathrm{S}}$ for the magnetopause. The same theoretical analysis suggests that these values correspond to $\sim 0.7$ of the peak-to-peak boundary displacement, thus consistently suggesting oscillation amplitudes of $\sim 2 R_{S}$ in both these cases. However, analysis of a few individual examples such as that shown in Figure 1 suggests that the amplitude can be significantly larger on occasion, possibly up to $4-5 \mathrm{R}_{\mathrm{S}}$ in the latter case, also in agreement with Clarke et al. [2010].

\section{Summary}

[23] In this report we have employed magnetic field and plasma electron data from 35 Cassini orbits during 20042007 on which the spacecraft traversed the outer region of Saturn's environment from the magnetosphere to the solar wind, to present a first study of magnetospheric period oscillations of Saturn's bow shock, following a study of re- lated magnetopause oscillations by Clarke et al. [2010]. The principal results are as follows.

[24] 1. The phasing of bow shock oscillation events within a timing window between 0.6 and 1.6 of the magnetospheric period is found to be significantly organized by the phase of the magnetic field oscillations observed inside the magnetosphere [Provan et al., 2009], thus showing the presence of bow shock oscillations at the magnetospheric period. The degree of organization is somewhat weaker than the corresponding effect for the magnetopause, with $74 \%$ of these events falling within the preferred $180^{\circ}$ Provan phase band between $60^{\circ}$ and $240^{\circ}$, compared with $89 \%$ for the magnetopause. The corresponding figures for the more restricted timing window between 0.6 and 1.0 of the magnetospheric period are $78 \%$ and $90 \%$.

[25] 2. The value of the Provan phase at the peak of the bow shock oscillation distribution is $133^{\circ} \pm 17^{\circ}$ for the timing window between 0.6 and 1.6 of the magnetospheric period, compared with $162^{\circ} \pm 15^{\circ}$ for the magnetopause within the same timing window (the latter value being essentially the same as that reported previously by Clarke et al. [2010]). These values are essentially equal to each other within the errors. The corresponding values for the timing window between 0.6 and 1.0 of the magnetospheric period are $140^{\circ} \pm 19^{\circ}$ for the bow shock and $144^{\circ} \pm 21^{\circ}$ for the magnetopause, again essentially equal. The bow shock and magnetopause thus oscillate in phase to within the established phase uncertainties of about $\pm 25^{\circ}$, thus implying signal propagation speeds across the magnetosheath of at least $\sim 100-200 \mathrm{~km} \mathrm{~s}^{-1}$. The values of the phase at the peak of the distributions indicate that the quasi-uniform field within the "core" region is rotated $\sim 130^{\circ}-160^{\circ}$ counterclockwise (as viewed from the north) of the instantaneous location of outward boundary excursion maxima.

[26] 3. Bow shock oscillations are commonly observed on Cassini orbits that cross the boundary region, with $\sim 60 \%$ of such orbits showing one or more oscillations within the timing window between 0.6 and 1.6 of the magnetospheric period and within the preferred $180^{\circ}$ phase sector from $60^{\circ}$ to $240^{\circ}$. These events are observed at all locations at which the boundary was encountered by the spacecraft, from 5 to 
$17 \mathrm{~h}$ in local time and between $-18^{\circ}$ and $+32^{\circ}$ in $\mathrm{KSM}$ latitude.

[27] 4. The data are consistent with the amplitudes of both bow shock and magnetopause oscillations being typically $\sim 1-2 \mathrm{R}_{\mathrm{S}}$, though evidence has also been presented of significantly larger values in the postnoon sector $\left(\sim 4-5 R_{S}\right)$ on occasion.

[28] A sketch summarizing these results and their relation to other oscillatory phenomena mentioned in section 1 is provided in Figure 6. This presents a view of the equatorial magnetosphere from the north with noon at the top and dusk to the left, showing the system at one particular phase of the rotating perturbations, when the maximum outward displacements of the magnetopause and bow shock occur near the noon meridian. The magnetopause and bow shock are shown by the blue and green lines, respectively, where the dot-dashed lines show the mean boundary positions, while the solid lines show the displaced boundaries. As discussed in more detail by Clarke et al. [2010], the plasma "bulge" in the outer magnetosphere reported by Burch et al. [2009] then also lies in the noon sector as indicated by the blue shaded area, while the high-density region of the Enceladus plasma torus in the inner magnetosphere described by Gurnett et al. [2007], shown by the dark green region, lies in the midafternoon sector, connected along disturbance phase fronts (red dashed lines) to the dayside plasma "bulge" and the outwardly displaced boundaries. The implication is that at this particular phase of the disturbance the total pressure in the outer magnetosphere is increased in the noon sector and decreased at midnight. Within the quasi-dipolar "core" magnetosphere bounded by the black dashed line, the equatorial perturbation magnetic field then points $\sim 140^{\circ}$ counterclockwise from the Sun as shown by the solid black arrows, in accordance with the results derived here. The maximum density in the inner plasma torus occurs in the local time sector where this field points in the positive azimuthal direction with respect to the planet, as shown. With increasing time this perturbation pattern then rotates counterclockwise around the planet at the magnetospheric period. SKR power output maximizes when the system rotates $\sim 70^{\circ}$ further counterclockwise from that shown, such that the field in the "core" region points $\sim 210^{\circ}$ counterclockwise from the Sun, radially outward in the postmidnight sector as indicated in section 1, and the plasma bulge and maximum outward boundary displacements lie in the late afternoon sector.

[29] We finally note that additional avenues of exploration of these phenomena are open for future studies, beyond those considered here. First, it can be seen from Figure 6 that the propagating perturbations in the boundary positions should also be accompanied by associated tilting of the boundary normals, directed eastward of the average as the boundary moves outward, and westward as it moves inward, associated with the eastward phase motion of the disturbance. We note that this is opposite to the behavior expected for Kelvin-Helmholtz disturbances propagating antisunward around the boundary in the dawn sector, but is in the same direction as the latter in the dusk sector. However, due to the very large length scales of the displacements along the boundaries (see Figure 6), these tilts are expected to be small, and may be difficult to detect in the presence of other shorter-scale phenomena. Second, the existence and nature of the inferred pressure perturbations in the outer magnetosphere remain to be investigated, for example the relative importance of perturbations in the plasma and magnetic field pressures in producing the boundary motions. Third, the overall physical origins of these oscillatory phenomena remain to be determined, of course, resulting in such highly coordinated rotating plasma and field perturbations throughout Saturn's magnetosphere.

[30] Acknowledgments. Work at Leicester was supported by STFC grant PP/E 000983/1. AJC and AM were supported by the STFC rolling grant to MSSL. We thank MK Dougherty and the Cassini team at Imperial College London for access to the processed magnetic field data, and GR Lewis and LK Gilbert for CAPS/ELS processing at MSSL. KEC was supported by a STFC Quota studentship.

[31] Amitava Bhattacharjee thanks Nicholas Achilleos and another reviewer for their assistance in evaluating this paper.

\section{References}

Achilleos, N., C. S. Arridge, C. Bertucci, C. M. Jackman, M. K. Dougherty, K. K. Khurana, and C. T. Russell (2008), Large-scale dynamics of Saturn's magnetopause: Observations by Cassini, J. Geophys. Res., 113, A11209, doi:10.1029/2008JA013265.

Andrews, D. J., E. J. Bunce, S. W. H. Cowley, M. K. Dougherty, G. Provan, and D. J. Southwood (2008), Planetary period oscillations in Saturn's magnetosphere: Phase relation of equatorial magnetic field oscillations and SKR modulation, J. Geophys. Res., 113, A09205, doi:10.1029/ 2007JA012937.

Andrews, D. J., S. W. H. Cowley, M. K. Dougherty, and G. Provan (2010), Magnetic field oscillations near the planetary period in Saturn's equatorial magnetosphere: Variation of amplitude and phase with radial distance and local time, J. Geophys. Res., doi:10.1029/2009JA014729, in press.

Arridge, C. S., N. Achilleos, M. K. Dougherty, K. K. Khurana, and C. T. Russell (2006), Modeling the size and shape of Saturn's magnetopause with variable dynamic pressure, J. Geophys. Res., 111, A11227, doi:10.1029/2005JA011574.

Burch, J. L., A. D. DeJong, J. Goldstein, and D. T. Young (2009), Periodicity in Saturn's magnetosphere: Plasma cam, Geophys. Res. Lett., 36, L14203, doi:10.1029/2009GL039043.

Burton, M. E., M. K. Dougherty, and C. T. Russell (2009), Model of Saturn's internal planetary magnetic field based on Cassini observations, Planet. Space Sci., 57, 1706-1713, doi:10.1016/j.pss.2009.04.008.

Carbary, J. F., D. G. Mitchell, S. M. Krimigis, D. C. Hamilton, and N. Krupp (2007a), Charged particle periodicities in Saturn's outer magnetosphere, J. Geophys. Res., 112, A06246, doi:10.1029/2007JA012351.

Carbary, J. F., D. G. Mitchell, S. M. Krimigis, and N. Krupp (2007b), Evidence for spiral pattern in Saturn's magnetosphere using the new SKR longitudes, Geophys. Res. Lett., 34, L13105, doi:10.1029/2007GL030167.

Carbary, J. F., D. G. Mitchell, P. Brandt, C. Paranicas, and S. M. Krimigis (2008), ENA periodicities at Saturn, Geophys. Res. Lett., 35, L07102, doi:10.1029/2008GL033230.

Clarke, K. E., et al. (2006), Cassini observations of planetary-period oscillations of Saturn's magnetopause, Geophys. Res. Lett., 33, L23104, doi:10.1029/2006GL027821.

Clarke, K. E., D. J. Andrews, C. S. Arridge, A. J. Coates, and S. W. H. Cowley (2010), Magnetopause oscillations near the planetary period at Saturn: Occurrence, phase, and amplitude, J. Geophys. Res., doi:10.1029/2009JA014745, in press.

Cowley, S. W. H., D. M. Wright, E. J. Bunce, A. C. Carter, M. K. Dougherty, G. Giampieri, J. D. Nichols, and T. R. Robinson (2006), Cassini observations of planetary-period magnetic field oscillations in Saturn's magnetosphere: Doppler shifts and phase motion, Geophys. Res. Lett., 33, L07104, doi:10.1029/2005GL025522.

Dougherty, M. K., et al. (2004), The Cassini magnetic field investigation, Space Sci. Rev., 114, 331-383, doi:10.1007/s11214-004-1432-2.

Dougherty, M. K., et al. (2005), Cassini magnetometer observations during Saturn orbit insertion, Science, 307, 1266-1270, doi:10.1126/science. 1106098.

Espinosa, S. A., D. J. Southwood, and M. K. Dougherty (2003), Reanalysis of Saturn's magnetospheric field data view of spin-periodic perturbations, J. Geophys. Res., 108(A2), 1085, doi:10.1029/2001JA005083.

Galopeau, P. H. M., and A. Lecacheux (2000), Variations of Saturn's radio rotation period measured at kilometer wavelengths, J. Geophys. Res., 105, 13,089-13,101, doi:10.1029/1999JA005089. 
Gurnett, D. A., et al. (2005), Radio and plasma wave observations at Saturn from Cassini's approach and first orbit, Science, 307, 1255-1259, doi:10.1126/science. 1105356

Gurnett, D. A., A. M. Persoon, W. S. Kurth, J. B. Groene, T. F. Averkamp, M. K. Dougherty, and D. J. Southwood (2007), The variable rotation period of the inner region of Saturn's plasma disk, Science, 316 , 442-445, doi:10.1126/science. 1138562

Gurnett, D. A., A. Lecacheux, W. S. Kurth, A. M. Persoon, J. B. Groene, L. Lamy, P. Zarka, and J. F. Carbary (2009), Discovery of a northsouth asymmetry in Saturn's radio rotation period, Geophys. Res. Lett. 36, L16102, doi:10.1029/2009GL039621.

Kurth, W. S., T. F. Averkamp, D. A. Gurnett, J. B. Groene, and A. Lecacheux (2008), An update to a Saturn longitude system based on kilometric radio emissions, J. Geophys. Res., 113, A05222, doi:10.1029/2007JA012861.

Masters, A., N. Achilleos, M. K. Dougherty, J. A. Slavin, G. B. Hospodarsky, C. S. Arridge, and A. J. Coates (2008), An empirical model of Saturn's bow shock: Cassini observations of shock location and shape, J. Geophys. Res., 113, A10210, doi:10.1029/2008JA013276.

Masters, A., N. Achilleos, C. Bertucci, M. K. Dougherty, S. J. Kanani, C. S. Arridge, H. J. McAndrews, and A. J. Coates (2009), Surface waves on Saturn's dawn flank magnetopause driven by the Kelvin-Helmholtz instability, Planet. Space Sci., 57, 1769-1778, doi:10.1016/j. pss.2009.02.010.
McAndrews, H. J., C. J. Owen, M. F. Thomsen, B. Lavraud, A. J. Coates, M. K. Dougherty, and D. T. Young (2008), Evidence for reconnection at Saturn's magnetopause, J. Geophys. Res., 113, A04210, doi:10.1029/ 2007JA012581.

Provan, G., D. J. Andrews, C. S. Arridge, A. J. Coates, S. W. H. Cowley, S. E. Milan, M. K. Dougherty, and D. M. Wright (2009), Polarization and phase of planetary-period magnetic field oscillations on high-latitude field lines in Saturn's magnetosphere, J. Geophys. Res., 114, A02225, doi:10.1029/2008JA013782.

Southwood, D. J., and M. G. Kivelson (2007), Saturn magnetospheric dynamics: Elucidation of a camshaft model, J. Geophys. Res., 112, A12222, doi:10.1029/2007JA012254.

Young, D. T., et al. (2004), Cassini plasma spectrometer investigation, Space Sci. Rev., 114, 1-112, doi:10.1007/s11214-004-1406-4.

D. J. Andrews, K. E. Clarke, and S. W. H. Cowley, Department of Physics and Astronomy, University of Leicester, Leicester LE1 7RH, UK. (kec10@ion.le.ac.uk)

A. J. Coates and A. Masters, Mullard Space Science Laboratory, University College London, Dorking RH5 6NT, UK. 